\title{
CLASSICAL AND QUANTUM ELECTRODYNAMICS AND THE B(3) FIELD
}


Editor-in-Chief: M. W. Evans (AIAS, Institute of Physics, Budapest, Hungary)

Associate Editors: S Jeffers (York University, Toronto)

D Leporini (University of Pisa, Italy)

J Moscicki (Jagellonian University, Poland)

L Pozhar (The Ukrainian Academy of Sciences)

S Roy (The Indian Statistical Institute)

Vol. 1 The Photon's Magnetic Field - Optical NMR Spectroscopy by $M$. W. Evans and F. Fahari

Vol. 2 Beltrami Fields in Chiral Media by $A$. Lakhtakia

Vol. 3 Quantum Mechanical Irreversibility and Measurement by P. Grigolini

Vol. 4 The Photomagneton and Quantum Field Theory: Quantum Chemistry, Vol. 1 by A. A. Hasanein and M. W. Evans

Vol. 5 Computational Methods in Quantum Chemistry: Quantum Chemistry, Vol. 2 by A. A. Hasanein and M. W. Evans

Vol. 6 Transport Theory of Inhomogeneous Fluids by L. A. Pozhar

Vol. 7 Dynamic Kerr Effect: The Use and Limits of the Smoluchowski Equation and Nonlinear Inertial Responses by J.-L. Dejardin

Vol. 8 Dielectric Relaxation and Dynamics of Polar Molecules by V. I. Gaiduk

Vol. 9 Water in Biology, Chemistry and Physics: Experimental Overviews and Computational Methodologies

by G. W. Robinson, S. B. Zhu, S. Singh and M. W. Evans

Vol. 10 The Langevin Equation: With Applications in Physics, Chemistry and Electrical Engineering by W. T. Coffey, Yu P. Kalmykov and J. T. Waldron

Vol. 11 Structure and Properties in Organised Polymeric Materials eds. E. Chiellini, M. Giordano and D. Leporini

Vol. 12 Proceedings of the Euroconference on Non-Equilibrium Phenomena in Supercooled Fluids, Glasses and Amorphous Materials eds. M. Giordano, D. Leporini and M. P. Tosi

Vol. 13 Electronic Structure and Chemical Bonding by J.-R. Lalanne

Vol. 14 Dialogues on Modern Physics by $M$. Sachs

Vol. 15 Phase in Optics by V. Perinova, A. Lukšs and J. Perina 
Vol. 16 Extended Electromagnetic Theory: Space Charge in Vacuo and the Rest Mass of the Photon by S. Roy and B. Lehnert

Vol. 17 Optical Spectroscopies of Electronic Absorption by J.-R. Lalanne, F. Carmona and L. Servant 
This page is intentionally left blank 


\section{CLASSICAL AND QUANTUM ELECTRODYNAMICS AND THE B(3) FIELD}

M W Evans \& L B Crowell

AlAS, Institute of Physics, Budapest, Hungary 


\section{Published by}

World Scientific Publishing Co. Pte. Ltd.

P O Box 128, Farrer Road, Singapore 912805

USA office: Suite 1B, 1060 Main Street, River Edge, NJ 07661

UK office: 57 Shelton Street, Covent Garden, London WC2H 9HE

\section{British Library Cataloguing-in-Publication Data \\ A catalogue record for this book is available from the British Library.}

\section{CLASSICAL AND QUANTUM ELECTRODYNAMICS AND THE B(3) FIELD}

Copyright (C) 2001 by World Scientific Publishing Co. Pte. Ltd.

All rights reserved. This book, or parts thereof, may not be reproduced in any form or by any means, electronic or mechanical, including photocopying, recording or any information storage and retrieval system now known or to be invented, without written permission from the Publisher.

For photocopying of material in this volume, please pay a copying fee through the Copyright Clearance Center, Inc., 222 Rosewood Drive, Danvers, MA 01923, USA. In this case permission to photocopy is not required from the publisher.

ISBN 981-02-4149-6 
Myron Evans dedicates this book to his wife Laura, and Lawrence Crowell dedicates it to his wife Elisheva, daughter MaryLynn and son Nathaniel. This book is also written in dedication to the recently deceased fathers of both authors. 
This page is intentionally left blank 


\section{Preface}

This text discusses the implications that electromagnetism has higher symmetries that may exist either at high energy or at low energy. In it there are illustrations of the possible consequences of this hypothesis. These implications involve the prospects for classical electrodynamics as well as the domain of extremely high energy physics up to the Planck scale. This possibility would illustrate that nature is more symmetrical in general and opens the prospect for a better understanding of nonlinear optical phenomenology and curious aspects of interferometry.

This extension of electromagnetism into a higher symmetry group illustrates that a number of concepts about physics, from classical electromagnetism to the theory of elementary particle physics may require revision if this extension is not sufficiently verified under experimental test. Physicists may then be required to revise a number of the current concepts if this extension is found to satisfy a body of supporting data. 
This page is intentionally left blank 


\section{CONTENTS}

Preface

Introduction

Chapter 1. Interaction of Electromagnetic Radiation with One Fermion

1.1. Lorentz Electron 3

1.2. Radiation Induced Electron and Proton Spin Rosonance 8

1.3. The $B^{(3)}$ Field 10

1.4. Electrodynamics as a NonAbelian Gauge Field Theory 10

1.5. Limitations of the $U(1)$ Theory 13

1.6. Classical Relativistic NonAbelian Electrodynamics 20

1.7. Relativistic Quantum Description 23

1.8. Nonrelativistic Quantum Description 24

1.9. Schrödinger Equation with Intrinsic Spin 26

1.10. Resonance Conditions in RFR 28

1.11. Chemical Shifts in NMR 30

1.12. Classical Derivation of the Inverse Faraday Effect 32 References 34

Chapter 2. The Field Equations of Classical $O(3)_{b}$ Electrodynamics 37

2.1. Introduction 37

2.2. The $O(3)_{b}$ Field Equations $\quad 37$

2.3. Basic S. I. Units 38

2.4. The $O(3)_{b}$ Vacuum Equations 39

2.4.1. The Coefficient $g$ in the Vacuum $\quad 39$

2.4.2. The $O(3)_{b}$ Field Tensor in the Vacuum 41

2.5. Reduction to Maxwell's Equations 47

2.6. The Fundamental Laws of $O(3)_{b}$ Electrodynamics 51

2.7. The Lorentz Force Equation in $O(3)_{b}$ Electrodynamics 54

2.8. Continuity Equation and Lorentz Condition in $O(3)_{b}$ Electrodynamics $\quad 56$

2.9. Primitive Concepts, Axioms and Constitutive Relations of $O(3)_{b}$ Electrodynamics $\quad 57$

2.10. The Inverse Faraday Effect 60

2.11. The effective $A^{(3)}$ Potential, Photoelectric and
Compton Effects and Radiation Reaction 
2.12. Summary 73

References $\quad 74$

Chapter 3. Origin of Electrodynamics in the General Theory of Gauge Fields 77

3.1. Closed Loop in Minkowski Space 77

3.2. Gauge Transformations 80

3.2.1. Gauge Transformation in the $O(3)_{b}$ Group $\quad 84$

3.3. The Sagnac Effect 86

3.4. Observation of $B^{(3)}$ in the Topological Phase 92

3.5. The NonAbelian Stokes Theorem and the

Electromagnetic Phase 102

3.6. Link between $B$ Cyclic Theorem and the NonAbelian

Stokes Theorem 109

References

Chapter 4. Nonlinear Propagation in $O(3)_{b}$ Electrodynamics:

Solitons and Instantons 115

4.1. Limitation in the $U(1)$ Theory 115

4.2. Identification of the Harmuth and $O(3)_{b}$

Field Equations

4.3. Structure of the $O(3)_{b}$ and Harmuth-Barrett Field Equations

4.4. Link between the $O(3)_{b}$ Equations and the Sine-Gordon Equations 122

4.5. Instantons 127

4.6. Higher Order Soliton Equations 132

References 136

Chapter 5. Physical Phase Effects in $O(3)_{b}$ Electrodynamics 137

5.1. Phase Effects 137

5.1.1. The Optical Ahronov-Bohm Effects 147

5.1.2. Phase Shift of the Inverse Faraday Effect 152

5.2. Phase Shift of the Optical Josephson Effect 155

5.3. Phase Factor of the Optical Hall Effect 156

References 156

Chapter 6. Quantum Electrodynamics and the $B^{(3)}$ Field 159

6.1. Introduction to Quantum Electrodynamics 159

6.2. A Brief Introduction to Differential Forms 168

6.3. The Physical Basis for NonAbelian Electrodynamics 170 
6.4. The Quantized $U(1)$ and $O(3)_{b}$ Electrodynamic Field 6.4.1. Numerical Results 6.4.2. Discussion of these Results

6.5. Quantum Electrodynamics of Elementary Scattering 206

6.6. Physics of Quantum Electrodynamics of Electrons and Photons with the $B^{(3)}$ Field

6.7. Nonrelativistic Estimate of the $B^{(3)}$ Contribution to the Lamb Shift

6.8. Derivation of the $1 / f$ Spectrum from NonAbelian Electrodynamics

6.9. Analogy from Classical Field to NonAbelian Quantum Electrodynamics References

Chapter 7. Quantum Chaos, Topological Indices and Gauge Theories

7.1. Introduction

7.2. Topological Number and Quantum Vortices

7.3. Density Operator Methods

7.4. Hamiltonian Chaotic Systems

7.5. Quantum Geometry and Bohm's Theory

7.6. Discussion and Problems

References

Chapter 8. Field Theory of $O(3)_{b} \mathrm{QED}$ and Unification with Weak and Nuclear Interactions

8.1. Discussion

8.2. Basics of Relativistic $O(3)_{b}$ QED

8.3. Renormalization of $O(3)_{b}$ QED

8.4. $\quad B^{(3)}$ Field as a Vacuum Symmetry

8.5.1. $S U(2) \times S U(2)$ Electroweak Theory with One Higgs Field

8.5.2. The $S U(2) \times S U(2)$ Extended Standard Model

8.5.3. Duality and Chiral Breaking

8.5.4. Discussion of the Theory, its Problems and their Remedies

8.6.1. Chiral and Vector Field in $S U(2) \times S U(2)$ 
8.6.3. Chiral and Vector Gauge Theories from Chiral Gauge Theories on the Physical Vacuum

8.6.4. The Occurrence of $O(3)_{b}$ Electrodynamics on the Physical Vacuum

8.6.4.1. The $S U(4)$ Model

8.6.5. Duality in Grand Unified Field Theory, and

Recent LEP1 Data

References

Chapter 9. Potential Application of $O(3)_{b}$ QED

9.1. Computation, Biophysics and $B^{(3)}$ Induced Entangled States

9.2. $\quad B^{(3)}$ Field and the Sequencing of DNA

Chapter 10. Duality and Fundamental Problems

10.1. Foundations for $S U(2)$ Electromagnetism

10.2. Rotations between Spacetime Conjugate Variables and Their Fluctuations in Quantum Gravity

10.3. Duality: Questions, Numerical Probes, and Quantum Uncertainty

10.4. Gravitation with One Killing Isometry

10.5. Brief Discussion on String Theory

10.5.1. Map between the String Surfaces and the Spacial 2-Surface

10.6. Overview of Conformal Groups

10.7. Conformal Structure of the Vacuum

10.8. Conformal Group and Gauge Theories According to Weighted Projective Spaces

10.9. Conformal Theory of $W_{\infty}$ String Vortices

10.10. Concluding Statements on Duality

References

Appendix 1 - Applications of Stokes' Theorem 